\title{
Neuropsychobiology 1993;28:222
}

\section{Author Index}

Alker, J. 87 Ambrus, A. 160 Appelberg, B. 192 Aschoff, A. 62

Bahner, E. 37 Bauer,J. 9 Becker, H. 82 Berger, M. 9 Boer, O. de 95 Bohus, B. 95 Brambilla, F. 187 Busted,J.M. 43

Cantell, C. 192 Clement, H.W. 91 Cools, |A.R. 100 Costelli, P. 177

Di Paolo, E. 177

Ellenbroek, B. 100 Engelen, S. 138

Felten, D.L. 110 Fett, C. 37 Fonzi, S. 177 Fritsch-Montero, R. 9

Gaillard, A.W.K. 72 Gallhofer, B. 17 Ganter, U. 9 Gemsa, D. 91 Georgi, R.V. 37 Gerrits, A. 138 Glatt, A.F. 118 Gruppe, H. 17 Guareschi-Cazzullo, A. 187

Hädicke, A. 87 Hasse, C. 91 Heijnen, C.J. 95 Hellhammer, D.H. 54, 76 Hennig, J. 5,37,82 Herrmann, W.H. 117 
Heymanns, J. 91 Hohagen, F. 9

Jacobs, R. 87 Jantscher, M. 17 Jibiki, I. 207

Katila, H. 192 Kirschbaum, C. 76 Kloet, E.R.de 100 Koolhaas, J.M. 95 Koopman, P. 122 Krieger, S. 9

Krijzer, F.N.C.M. 117, 122 Kubota, T. 207

Lancel, M. 154 Laschefski, U. 82 Lehmann, D. 212 Lehnert, H. 54

Maeda, T. 207 Mann, K. 54 Michel, CM. 212 Murialdo, G. 177 Musetti, C. 187

Naukkarinen, H. 192 Netter, P. 5, 37, 82 Nink, M. 54 Nobile, P. 187

Olfff, M. 66 Olivier, B. 122 Opper, Cl 37

Pirke, K.-M. 76 Pohlner, K. 91 Polleri, A. 177 Porro, S. 177 Praag, H!M. van 197 Pröhl, F. 87

Rammsayer, T. 5, 37, 82 Richter, S. 87 
Riezen, H. van 118 Rimón, R. 192 Röschke, J. 54 Roth, J. 106 Rots, N.Y. 100 Roy, A. 184 Ruigt, G.S.F. 138

Sabel, B.A. 62 Sarkadi, A. 160 Schäfer, F. 91 Schedlowski, M. 87 Schmidt, R.E. 87 Schweiger, E. 25 Solinas, G. 177 Stemmler, G. 47 Stratmann, G. 87 Szporny, L. 160 Tacchini, C. 187 Tewes, U. 87 Timmer,J. 9 Torre, F. 177 Tosca, P. 177

Ursin, H. 66

Veltman, J.A. 72 Verbon, F. 138

Wagner, T.O.F. 87 Wahlbeck, K. 192 Warburton, D.M. 43 Weiner, N. 91 Wesemann, W. 37,91 Weyerbrock, A. 9 Wittling, W. 25

Yamaguchi, N. 207

Zeisberger, E. 106 Zerbi, F. 177 Zuckerman, M. 30 